the telescope, suspended by its centre, must not bend more than a millimetre in the most unfavourable positions, according to the calculations by which $\mathrm{M}$. Wolf determines its dimensions. Experience has verified his calculations; the two mirrors remain exactly centred upon each other in all positions of the telescope.

It is necessary, however, to be able to direct the tube toward any point of the sky, and it is necessary, moreover, that when the star is once in the field of the instrument, that should be able to follow it, by a simple movement, in its apparent course through the heavens. This is accomplished by what is called the equatorial mounting of the telescope. This revolves on an axis, cast of iron and steel, whose direction is parallel to the axis of the celestial sphere. Then it may be inclined more or less on this axis, by turning round in a second axis of steel, which crosses the former at a right angle, and partakes in its movement of rotation. The whole of the arrangement of double axes, a veritable wonder of mechanism, from the precision and ease of the movement, weighs with the telescope 10,000 kilogrammes. Such is the mass which, like the hand of a gigantic chronometer, must follow with precision the march of the stars in the vault of heaven, obedient to the action of clockwork, controlled by a Foucault regulator.

To realise this wonder, M. Eichens had to put together the most delicate apparatus of the mechanics of precision, and, preserving their delicacy intact, give them the strength necessary to support great weights. We cannot explain in detail the series of these wonders, tell how friction is almost annihilated throughout, how all the parts are in equilibrium, whatever be the position of the telescope: how, in fine, at the same time that the instrument follows the movement of the sky, the observer may at his pleasure move it with perfect ease in all directions by means of contrivances placed at his hand.

The perfection of mechanism would be nothing if it did not serve the purpose of observing the stars with an optical apparatus of equal perfection. Let us at once say that the first attempts which have been made with the instrument have completely satisfied the astronomers. Not only has the mirror acquired, under the hand of $M$. Martin, the rigorously parabolic form which gives it the property of collecting in a single point the rays of a star, but the very complex eye-piece, by means of which the luminous point is observed, is itself without a single defect. It now only remains to silver the surface of the mirror, an operation at present easy, by the processes of M. Ad. Martin, and which will be accomplished in a large dish I'30 metres in diameter. Meantime, the surface of the polished glass reflects sufficient light to make it possible to observe the most feeble stars; directed towards the moon, the telescope concentrates in the eye a light almost intolerable. It may be judged from this what will be the brilliancy of celestial images when the silvered mirror will throw upon the eye, not merely scarcely one-half, but more than nine-tenths of the light which it receives.

The comparison which we made above between the Marseilles telescope of 0.80 metre aperture and the most powerful instruments elsewhere, allows us to predict the results which science has a right to expect from a telescope whose mirror is greater by half, and whose mechanism has reached the latest limits of perfection. M. Wolf, to whom the use of the telescope has been entrusted, proposes to employ it in observing the planets and their satellites. At the same time the new telescope will be fitted with all the apparatus necessary for photography and the spectroscopic observation of the stars. It should be remembered, however, that the use of such a gigantic instrument requires a long apprenticeship; the Melboume instrument had two observers before it came into the hands of an astronomer who knew how to make good use of it.
In a few weeks will be completed the first of the great instruments promised to France by MM. Le Verrier and Foucault. The construction of the telescope has been undertaken first, that it may serve as a study for the construction, much more delicate, of the great refractor of I6 metres in length, with an object-glass of 75 metre aperture. The success of the reflector is a guarantee that M. Le Verrier, with his eminent colleagues, will accomplish satisfactorily the second part of his great and patriotic enterprise.

\section{THE LOAN EXHIBITION OF SCIENTIFIC \\ APPARATUS AT SOUTH KENSINGTON}

$\mathrm{N}$ the 3 rd inst., as we have already intimated, Her Imperial Highness the Crown Princess of Germany invited to her palace forty of the representatives of science of Berlin, to lay before them the plan of the London Exhibition of Scientific Apparatus, and to ask their co-operation for this purpose. Amongst those honoured by invitations were the Ministers of Education and of Commerce, the Postmaster-General, and the following professors of the University :- $M M$. Braun (botanist), Dove, Helmholtz, and Kirchhoff (physicists), Du Bois-Reymond (physiologist), Kiepert (geographer), Förster (astronomer), Peters (zoologist), Kronecker (mathematician), Websky (mineralogist), Hofmann, Oppenheim, and Sell (chemists), Wichelhaus (technologist). Orta (agriculturist); the following professors of the Polytechnic School :-MM. Reulaux (mechanician), Liebermann (chemist), Vogel (photographer), and Scheibler (agricultural chemist); the director of the South Kensington Museum, Mr. Cunliffe-Owen, the directors of the German Industrial Museum, MM. Gruner and Lessing, the manufacturers, Dr. Werner Siemens (member of the Academy), and Dr. Martius. The illustrious hostess, as well as His Imperial Highness the Crown Prince, pleaded warmly for the worthy representation of Germany in the London Exhibition. Although the short time left for preparation, and the coincidence of the exhibition with that of Philadelphia, were generally felt as serious drawbacks, some of the men of science present taking the lead assembled the following day, when a general committee was formed under the presidency of Dr. A. W. Hofmann ; with the view of forming special committees for the different branches of the exhibition, and of inviting one member of every German University and Polytechnic School to co-operate with them. An invitation to the men of science and the manufacturers of scientific apparatus has already been issued.

"Science," the invitation says, "being the common property of all nations, the exhibition of the appliances by which it is promoted partakes of an international character. The objects pursued by the English Cormmission in organising the Exhibition have in Germany also been recognised as worthy of attainment, and, in order to give an impulse in our Fatherland to German participation in the Exhibition, a Committee has been formed, at the special instance of the Crown Prince and Princess of Germany, which has been intrusted by the English Commission with the collection and selection of objects worthy of being exhibited."

The invitation then proceeds to detail the conditions of exhibition settled by the Science and Art Department, and concludes as follows:-

"The Exhibition of Scientific Apparatus in London essentially differs from the former Exhibitions, as it pays less regard to merely commercial interests, but keeps in view the higher aim of disseminating as widely as possible the knowledge of the different methods of science. In order to render full justice to this task, the British Governrnent (Science and Art Department) will bear all the c ssts of packing, sending, and returning any objects that may be confided to its care." 
The Minister of the Admiralty has promised to send every instrument which should appear suitable for the purpose. The Committee of the German Chemical Society have elected superintendents to procure a worthy representation of chemical apparatus and specimens of chemical compounds of scientific interest. It has been decided to address an invitation to the members of the society to send such specimens to Berlin, in order to form a systematical and uniform collection of rare and interesting chemicals.

We may state generally that the Governments of Belgium, France, Germany, Italy, the Netherlands, and Switzerland, have now appointed committees to act in concert with the general committee in London. The Government of the United States has, through Mr. Fish, intimated that it is in communication with the various Departments and Scientific Institutions, with the object of forwarding the Exhibition.

\section{BEEATS IN MUSIC *}

II.

THE second kind of beat differs from the first in that it arises from the imperfection, not of unisons, but of wide-apart consonances, such as the third, fourth, fifth, sixth, and octave.

This beat is well known practically to organ tuners, and may be soon rendered appreciable to any musical ear. Taking the fifth as an example, ict the two notes $\frac{a}{(4)}=1$ be sounded on an organ, or any instrument of sustained tones. If they are perfectly in tune, the united sound will be smooth and even; but if one of them be sharpened or flattened a little, a beat will be heard just as in the case of the imperfect unison; and which, like it, will increase in rapidity as the note is made more and more out of tune. That this is not the same beat as Tartini's is obvious from the failure of the rule for the latter when applied to the former; for example, when the concord is in tune the upper note vibrates 768 , and the lower one 512 vibrations per second, therefore there ought to be 256 beats per second; but in reality there are no beats at all, they only begin when the notes are put out of tune; hence the Tartini-beat rule is useless and inapplicable in this case.

This beat may be called the consonance beat, and it has also been termed "Smith's beat," from its having been first investigated by him.

The theory of Smith's beat, as given by Smith himself, is complicated and difficult to describe ; but we will endeavour to give some idea of its nature and cause.

We must return to the illustration of the coffin-makers, Suppose one of them to have sold his business to another man in the trade, who was so much more active and energetic that he could drive his nails half as fast again as ordinary workmen. Call him $A$, and suppose that when he began to work it was found that he struck exactly three blows to two of his neighbour B. As B struck roo per minute, A will now strike 150. And assume that on a certain day they both begin exactly together. The passer-by will hear that every third blow of A exactly coincides with every second of $B$; so that he will notice fifty coincidences in a minute; or to describe them more correctly, he will notice per minute fifty phases of compound effects, in each of which there is a coincidence. This phase constitutes Tartini's beat, but now very much augmented in rapidity from what it was before: then there were only one or two coincidences per minute, now there are fifty.

Now suppose that A, from some slight exhilarating

* By W. Pole, F.R.S., Mus. Doc. Oxon. Continued from p. 2x4. cause, begins to strike a little faster; i.e. that he makes $15 \mathrm{I}$ blows in a minute instead of 150 . Let us endeavour to find out what will be the result on the listener. Still supposing the two strokes to begin withi a coincidence, the third blow of A will still coincide very nearly indeed with the second of $B$; it will only differ from it by $\frac{7}{7500}$ of a minute, a quantity inappreciable to the ear. Hence the Tartini phase will at this time be practically unharmed. But after a few repetitions the divergence of the blows will be so great as to become appreciable, and the listener will begin to notice a series of changes of form of the Tartini phase, in which there is now no coincidence of the blows, but only a variation of their arrangement, which, moreover, is itself constantly varying. After a time, however, these changes will exhaust their possible varieties, the listener will notice that two of the blows begin to approach again, and at last will coincide, as they did before. He thus notices a long cycle of the Tartini beats, and this long cycle is the Smith's beat. It is, in fact, a beat of what mathematicians would call the second order; the first, or Tartini's beat, is a cycle of differing periods; the second, or Smith's beat, is a cycle of differing cycles.

Let us next attempt a numerical estimation of the length of this second cycle in the case of the coffinmakers. To effect this we must inquire when the coincidences of two blows will recur. It is plain that they will recur at the end of the minute, i.e. if the first blow of A coincided with the first of $B$, then the I5 Ist of $A$ will coincide with the rooth of B. This will give one long cycle, or one Smith's beat, per minute. A careful comparison of the times of the respective blows will show, moreover, that (since 100 and 15 I are prime to each other) there will be no other exact coincidence during the minute; and a hasty reasoner may conclude that one beat per minute will be the proper number. But if the listener be asked to describe what he hears, he will dissent from this and say confidently that there are two places in the minute where he hears a coincidence. To test his asser. tion, let us apply Young's principle mentioned before, and inquire whether in the course of the minute there is any other place where the blows so nearly coincide that the ear may mistake them for real coincidences. The 74 th blow of A will occur at $\frac{74}{5}$ of a minute after starting, whereas the 49 th blow of $B$ will occur at $\frac{49}{100}$ of a minute. The difference between these is only 15 tōo of a minute, which is quite inappreciable. Hence, practically, there will be two parts of the minute where the blows coincide, and there will be consequently two Smith's beats in the same time.* If we were to suppose A to make I 52 blows per minute (or 148 , for a deficiency would produce the same result as an excess) to B's Ioo, we should, calculating on the above plan, find four cycles or beats per minute. Or we may alter the proportion: suppose for example, A, intending to make five blows to B's four, makes really 126 per minute instead of 125 as he ought to do ; it will be found by calculation that there will be four places of coincidence in the minute, or four Smith's beats; if he strikes 127 blows, there will be eight Smith's beats-and so on.

We hope the foregoing homely illustration will help to render clear the nature of the Smith's beat as applied to sounds. Although the Tartini beat may not b? really converted, as Young supposed, into the Tartini harmonic, but, according to Helmholtz, remains as a beat, inappreciable by reason of its great rapidity, it certainly has a physical and mathematical existence; and it as certainly changes its phases by reason of the small divergence of the times of vibration from those due to the true concord, and it is the recurrence of similar phases in a long cycle

* Mr. De Morgan, in his admirable paper elucidating Smith's profound investigation, unfortunately omits to notice this important element of the approximate coincidences. The consequence is that his explanation is not easy to follow, and indeed would appear wrong, although his results are perfectly correct. 